\title{
Color Glass Condensate at NLO: phenomenology at HERA, RHIC and the LHC
}

\author{
Javier L. Albacete \\ Institut de Physique Theéorique CEA/Saclay, F-91191 Gif-sur-Yvette Cedex, FRANCE.
}

DOI: http://dx.doi.org/10.5689/UA-PROC-2010-09/40

\begin{abstract}
A brief review of the phenomenological studies in the field of heavy ion collisions based on the Color Glass Condensate theory and, in particular, of those relying in the use of the BK equation including running coupling effects is presented.
\end{abstract}

\section{Introduction}

The Color Glass Condensate effective theory provides a consistent framework to study QCD scattering at high energies (for a review see e.g. [1, 2]). The main physical ingredient in the CGC is the inclusion of unitarity effects through the proper consideration of non-linear recombination effects, both at the level of particle production and also in the quantum evolution of hadronic wave functions. Such effects are expected to be relevant when nuclei (or hadrons, in full generality) are proven at small enough values of Bjorken- $x$. In that regime gluon occupation numbers are very large and gluon self-interactions become highly probable, thus taming, or saturating, further growth of the gluon densities. While the need for unitarity effects comprised in the CGC is, at a theoretical level, clear, the real challenge from a phenomenological point of view is to assess to what extent they are present in available data. Such is a difficult task, since different physical mechanisms concur in data, and also because the limit of asymptotically high energy in which the CGC formalism is developed may not be realized in current experiments. In that sense, the calculation of higher order corrections to the CGC formalism has supposed important leap forward in sharpening the CGC as an useful phenomenological tool.

\section{Running coupling Balitsky-Kovchegov equation.}

The leading order BK-JIMWLK equations resums soft gluon emission in the leading logarithmic (LL) approximation in $\alpha_{s} \ln 1 / x$ to all orders, besides of including non-linear terms required by unitarity. At such degree of accuracy, the theory is incompatible with data. Such insufficiency of the theory has been partially fixed by the calculation of running coupling corrections to the BK-JIMWLK equations through the inclusion of quark loops to all orders [4, 5]. Among other interesting dynamical effects, running coupling effects tame the growth of the saturation scale down to values compatible with experimental data [6]. Due to the complexity of the JIMWLK equations, in phenomenological works it is more feasible to solve the BK equation, 
more tractable numerically, which corresponds to their large- $N_{c}$ limit. It reads

$\frac{\partial \mathcal{N}(r, Y)}{\partial Y}=\int d^{2} \mathbf{r}_{1} K^{\mathrm{run}}\left(\mathbf{r}, \mathbf{r}_{1}, \mathbf{r}_{2}\right)\left[\mathcal{N}\left(r_{1}, Y\right)+\mathcal{N}\left(r_{2}, Y\right)-\mathcal{N}(r, Y)-\mathcal{N}\left(r_{1}, Y\right) \mathcal{N}\left(r_{2}, Y\right)\right]$

where $\mathcal{N}(r, Y)$ is the dipole scattering amplitude on a dense target, $Y=\ln x_{0} / x$ the rapidity, $r$ the dipole transverse size and $r_{2}=r-r_{1}$. It turns out that running coupling effects can be incorporated to the evolution equation through just a modification of the evolution kernel, referred to as $K^{\text {run }}$ in Eq. (1) (see [6] for an extended discussion on the subject) Finally, Eq. (1) needs to be suplemented with initial conditions, which can be choosen to be of the McLerranVenugopalan type [7]. This introduces two free parameters: The value $x_{0}$ where the evolution starts and the initial saturation scale $Q_{0}$. Finally, the unintegrated gluon distribution entering the different production processes discussed below is related to the dipole amplitude in Eq. (1) through a Fourier transform (see Eq. (5)). In all the phenomenological works described below those two parameters are fitted to experimental data.

\section{Structure functions at HERA}

Data on inclusive structure functions in $\mathrm{e}+\mathrm{p}$ collisions at small- $x$ performed in HERA provide a good ground to test the CGC. According to the dipole model formulation of deep inelastic scattering, the $\gamma^{*}-p$ cross section can be written as

$$
\sigma_{T, L}\left(x, Q^{2}\right)=2 \sum_{f} \int_{0}^{1} d z \int d \mathbf{b} d \mathbf{r}\left|\Psi_{T, L}^{f}\left(e_{f}, m_{f}, z, Q^{2}, \mathbf{r}\right)\right|^{2} \mathcal{N}(\mathbf{b}, \mathbf{r}, x),
$$

where $\Psi$ describes the wave function for the virtual photon to split in a $q \bar{q}$ pair and $\mathcal{N}$ is the dipole scattering. Fig 1. shows a fit [8] to data on the reduced cross section measured at HERA using rcBK equation to describe the $x$-dependence of the dipole scattering amplitude in Eq. (2). Such good agreement with data suggest the possible presence of saturation effects as described in the rcBK equation.

\section{Single inclusive particle production and nuclear modi- fication factors}

Nuclear effects in $\mathrm{p}+\mathrm{A}$ or $\mathrm{A}+\mathrm{A}$ collisions are typically evaluated in terms of the nuclear modification factors:

$$
R_{p A}=\frac{\frac{d N^{p A}}{d y d^{2} p_{t}}}{N_{\text {coll }} \frac{d N^{p p}}{d y d^{2} p_{t}}}
$$

where $N_{\text {coll }}$ is the number of collisions. If high-energy nuclear reactions were a mere incoherent superposition of nucleon-nucleon collisions, then the observed $R_{p A}$ should be equal to unity. However, RHIC measurements in $\mathrm{d}+\mathrm{Au}$ collisions (or peripheral $\mathrm{Au}+\mathrm{Au}$ collisions) $[12,13]$ in the forward rapidity region exhibit a clear suppression for all experimentally accesible values of $p_{t}$. . However, at more forward rapidities such Cronin enhancement disappears, turning into an almost homogeneous suppression for all the measured values of $p_{t}$. According to $2 \rightarrow 1$ kinematics, the $x$-values probed in the projectile and target are $x_{1(2)}=\left(m_{t} / \sqrt{s}\right) e^{ \pm y}$. Thus, 


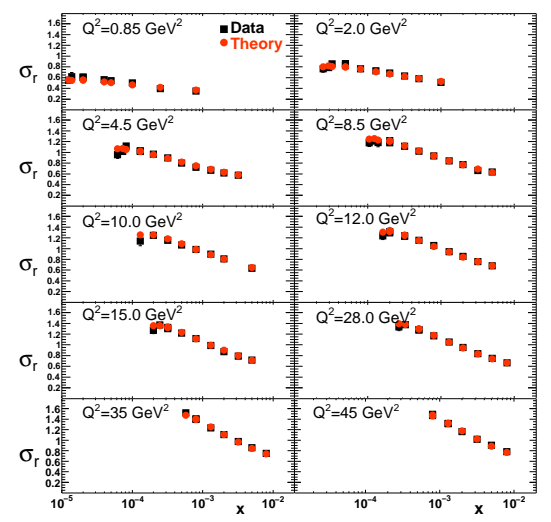

Figure 1: Fits to data on the reduced cross section $\sigma_{r}$ measured in e+p scattering at HERA.

$x$-values are small for $y>1$ at RHIC energies, offering a cleaner opportunity to explore CGC effects. There, the CGC formulation of single particle production takes on a relatively simple form [14]:

$$
\begin{array}{r}
\frac{d N_{h}}{d y_{h} d^{2} p_{t}}=\frac{K}{(2 \pi)^{2}} \sum_{q} \int_{x_{F}}^{1} \frac{d z}{z^{2}}\left[x_{1} f_{q / p}\left(x_{1}, p_{t}^{2}\right) \tilde{N}_{F}\left(x_{2}, \frac{p_{t}}{z}\right) D_{h / q}\left(z, p_{t}^{2}\right)\right. \\
\left.+x_{1} f_{g / p}\left(x_{1}, p_{t}^{2}\right) \tilde{N}_{A}\left(x_{2}, \frac{p_{t}}{z}\right) D_{h / g}\left(z, p_{t}^{2}\right)\right]
\end{array}
$$

where $p_{t}$ and $y_{h}$ are the transverse momentum and rapidity of the produced hadron, and $f_{i / p}$ and $D_{h / i}$ refer to the parton distribution function of the incoming proton and to the final-state hadron fragmentation function respectively. Thus, in the forward region the projectile is in the dilute regime and characterized by its parton distribution functions, while the nucleus is deep in the saturation region and characterized by unintegrated gluon distributions taken from the solutions of the rcBK equation:

$$
\tilde{N}_{F(A)}(x, k)=\int d^{2} \mathbf{r} e^{-i \mathbf{k} \cdot \mathbf{r}}\left[1-\mathcal{N}_{F(A)}\left(r, Y=\ln \left(x_{0} / x\right)\right)\right],
$$

where $k$ refers to transverse momentum. With this set up we reach a very good description of forward neutral pions and negatively charged hadrons yields as measured by the STAR and BRAHMS Collaborations respectively in $\mathrm{d}+\mathrm{Au}$ minimum bias and in $\mathrm{p}+\mathrm{p}$ collisions, as shown in Fig 2. All the details of the calculation and fit parameters can be found in [15].

By simply taking the ratios of the corresponding spectra, we get a very good description of the nuclear modification factors at forward rapidities. It should be noted that we use the same normalization as the experimentalist do in their analyses of minimum bias $\mathrm{d}+\mathrm{Au}$ collisions, i.e. we fix $N_{\text {coll }}=7.2$. Physically, the observed suppression is due to the relative enhancement of non-linear terms in the small- $x$ evolution of the nuclear wave function with respect to that of a proton. However, it has been argued that the observed suppression at forward rapidities is not an effect associated to the small values proven in the nuclear wave function but, rather, 

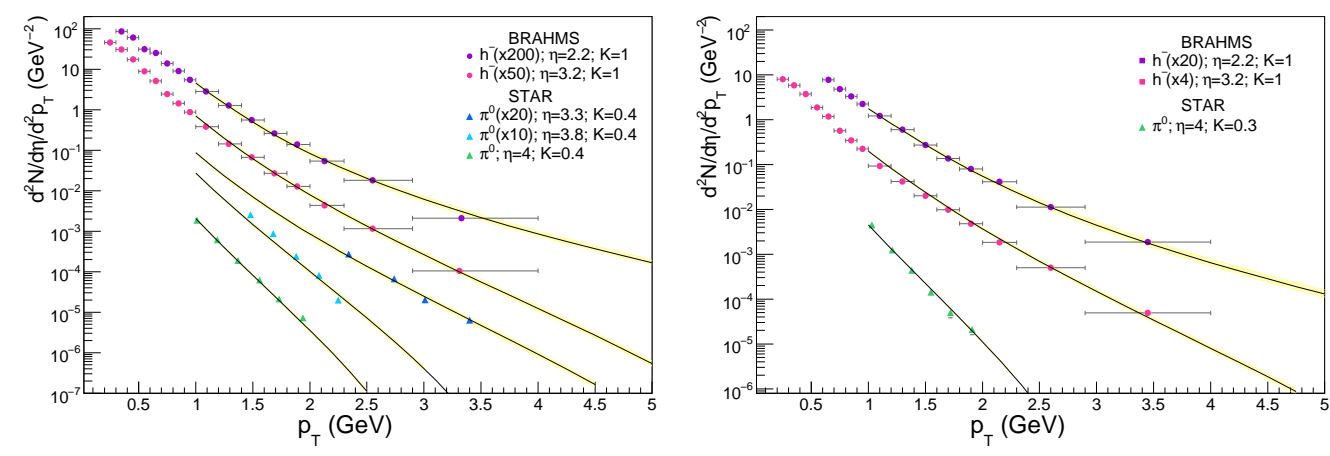

Figure 2: Negative charged hadron and neutral pions at forward rapidities measured by the BRAHMS [12] and STAR [13] Collaborations in $\mathrm{p}+\mathrm{p}$ (left) and $\mathrm{d}+\mathrm{Au}$ minimum bias (right) collisions compared to our calculation [15].

to energy-momentum conservation corrections relevant for $x_{F} \rightarrow 1$ Such corrections are not present in the CGC, built upon the eikonal approximation. Thus, the energy degradation of the projectile parton through either elastic scattering or induced gluon brehmstralung would be larger in a nucleus than in proton on account of the stronger color fields of the former, resulting in the relative suppression observed in data. A successful description of forward ratios based on the energy loss calculation was presented in [16].

Thus, at the level of only this observable, one is in an inconclusive situation. Disentangling the underlying dynamics of the suppression requires either data at larger energies or, alternatively, the study of more exclusive observables. The huge leap forward in collision energy reached at the LHC allows for an exploration of small- $x$ effects already at mid-rapidity. There, both the target and projectile $x$ are small, and energy loss effects associated to large- $x_{F}$ effects are expected to be small. CGC predictions for light hadrons $R_{p} P p$ agree to predict a sizable suppresion $\sim 0.6$ at LHC energies already at mid-rapidity.

\section{Forward di-hadron correlations}

As said before, disentangling CGC from other dynamical effects demands the analysis of more exclusive observables. The opportunity is provided by the recent measurement of forward dipions correlations in $\mathrm{d}+\mathrm{Au}$ collisions by the STAR collaboration [17]. The experimental data for the coincidence probability $C P(\Delta \phi)$ feature a clear suppression of the away-side correlations with respect to the ones observed $\mathrm{p}+\mathrm{p}$ collisions. This suppression is naturally explained in the CGC: The two pions experimentally observed originate from a valence quark-gluon system in perfect back-to-back correlation. The quark-gluon system is put on-shell through the interaction with the nucleus, as a result of which the quark and gluon also acquire a transverse momentum of the order of the saturation scale of the nucleus. When that scale, which marks the onset of non-linear effects, is comparable to the initial transverse momenta of the quark and gluon, their intrinsic angular correlation is washed out. Finally, the outgoing quark and gluon fragment independently into hadrons. A recent calculation CGC [18] provides a very good comparison with available data. There, the required information on the nuclear wave function is built, 


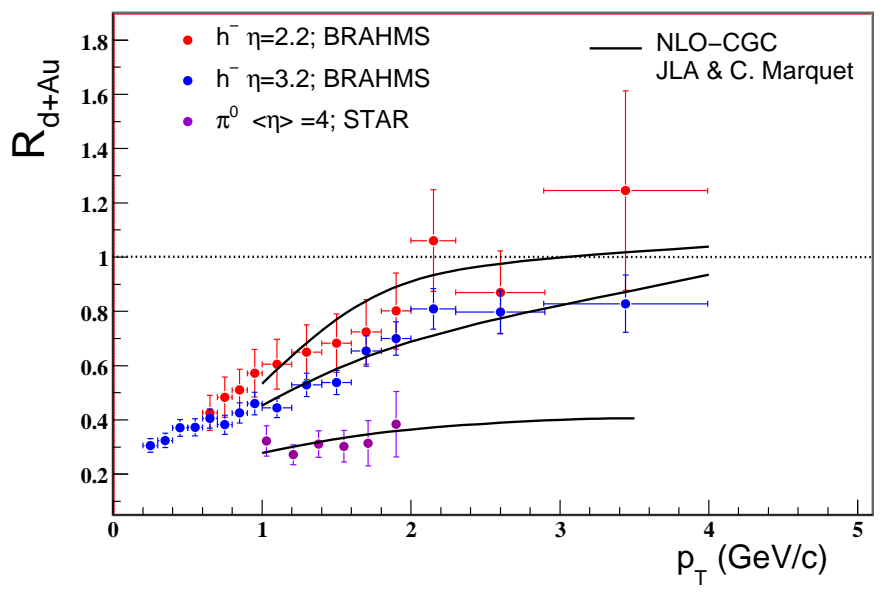

Figure 3: Nuclear modification factors at forward rapidities in minimum bias $\mathrm{d}+\mathrm{Au}$ collisions in the CGC [15].

through the use of the gaussian approximation, from the two-point function Eq. (5) constrained by the analyses of single inclusive spectra, making it a parameter-free calculation. This study provides, arguably, the most compelling evidence for the presence of CGC effects in available data.

\section{Acknowledgments}

I would like to thank the organizers of the ISMD 2010 symposium for their invitation to such a nice meeting. This work is supported by a Marie Curie Intra-European Fellowship (FP7PEOPLE-IEF-2008), contract No. 236376.

\section{Bibliography}

\section{References}

[1] Gelis F, Iancu E, Jalilian-Marian J and Venugopalan R 2010 (Preprint 1002.0333)

[2] Weigert H 2005 Prog. Part. Nucl. Phys. 55 461-565 (Preprint hep-ph/0501087)

[3] Albacete J L, Armesto N, Milhano J G, Salgado C A and Wiedemann U A 2005 Phys. Rev. D71 014003 (Preprint hep-ph/0408216)

[4] Kovchegov Y and Weigert H 2007 Nucl. Phys. A 784 188-226 (Preprint hep-ph/0609090)

[5] Balitsky I I 2007 Phys. Rev. D 75014001 (Preprint hep-ph/0609105)

[6] Albacete J L and Kovchegov Y V 2007 Phys. Rev. D75 125021 (Preprint arXiv:0704. $0612[\mathrm{hep}-\mathrm{ph}])$ 


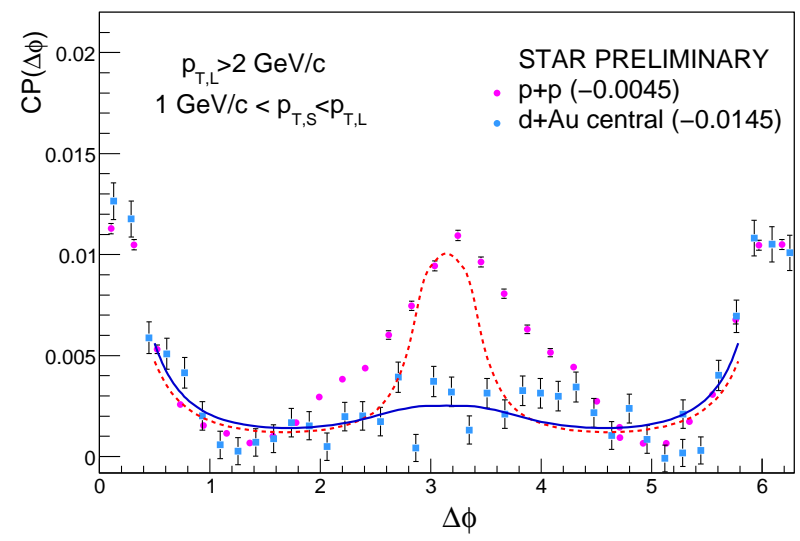

Figure 4: Comparison of the CGC calculation of [18] with data for the coincidence probability at forward rapidities in $\mathrm{d}+\mathrm{Au}$ (blue) and $\mathrm{p}+\mathrm{p}$ collisions (red).

[7] McLerran L D and Venugopalan R 1998 Phys. Lett. B424 15-24 (Preprint nucl-th/ 9705055)

[8] Albacete J L, Armesto N, Milhano J G, Quiroga-Arias P and Salgado C A 2010 (Preprint 1012.4408)

[9] Kharzeev D, Levin E and Nardi M 2005 Nucl. Phys. A747 609-629 (Preprint hep-ph/ 0408050)

[10] Albacete J L 2007 Phys. Rev. Lett. 99262301 (Preprint 0707.2545 )

[11] Back B B et al. 2005 Nucl. Phys. A757 28-101 (Preprint nucl-ex/0410022)

[12] Arsene I et al. (BRAHMS) 2004 Phys. Rev. Lett. 93242303 (Preprint nucl-ex/0403005)

[13] Adams J et al. (STAR) 2006 Phys. Rev. Lett. 97152302 (Preprint nucl-ex/0602011)

[14] Dumitru A, Hayashigaki A and Jalilian-Marian J 2006 Nucl. Phys. A765 464-482 (Preprint hep-ph/0506308)

[15] Albacete J L and Marquet C 2010 Phys. Lett. B687 174-179 (Preprint 1001.1378)

[16] Kopeliovich B Z, Nemchik J, Potashnikova I K, Johnson M B and Schmidt I 2005 Phys. Rev. C72 054606 (Preprint hep-ph/0501260)

[17] Braidot E and collaboration f t S 2010 (Preprint 1005.2378)

[18] Albacete J L and Marquet C 2010 (Preprint 1005.4065) 